\title{
Transitivity properties of ternary fuzzy relations
}

\author{
Lemnaouar Zedam ${ }^{a}$ and Bernard De Baets ${ }^{b}$ \\ ${ }^{a}$ Laboratory LMPA, Department of Mathematics, University of M'sila, \\ P.O. Box 166 Ichbilia, Msila 28000, Algeria, lemnaouar.zedam@univ-msila.dz \\ ${ }^{b}$ KERMIT, Department of Data Analysis and Mathematical Modelling, Ghent University, \\ Coupure links 653, B-9000 Gent, Belgium, Bernard.DeBaets@ugent.be
}

\begin{abstract}
Recently, we have introduced six types of composition of crisp ternary relations. These compositions are close in spirit to the definition of the composition of binary relations. In this note, we extend these notions to the fuzzy setting. Based on these types of composition, we introduce several types of transitivity of a ternary fuzzy relation and investigate their properties.
\end{abstract}

Keywords: Fuzzy ternary relation, relational compositions, Transitivity.

\section{Introduction}

One of the most important properties of binary relations is the transitivity property. The classical concept of transitivity of relations is generalized in fuzzy set theory by the $*$-transitivity property of fuzzy relations, where $*$ is a t-norm [23]. In addition to its role as an interesting condition of some useful classes of (fuzzy) binary relations (e.g., preorder relations, order relations, equivalence relations), it is an essential tool in many fields of application. For instance, in the fields of (fuzzy) preference modelling and multi-criteria decision making $[6,11,13]$ and in fuzzy control [17].

Using the definition of composition of relations, the transitivity property can be formulated more concisely, such that a (fuzzy) binary relation $R$ is transitive if and only if $R \circ R \subseteq R$. Algorithms that search for the transitivity property can be used in many approximate reasoning applications, including database management systems (DBMS), pattern recognition, expert systems, artificial intelligence (AI), and intelligent systems.

In recent years, the interest in ternary relations is on the rise, as they play an important role in many theoretical and applied areas. From a theoretical point of view, ternary relations have been studied in mathematics (e.g., in algebra [7, 19], in (fuzzy) triadic formal concept analysis $[5,14,18]$ and in logic [21]). In applications, (fuzzy) ternary relations can be found in many different areas, for instance in social sciences (e.g., philosophy [3]), in biology (e.g., modelling of phylogenies [28]), in computer science (e.g., the Resource Description Framework (RDF) [27]). Some classes of ternary relations came to play an important role in specific applications, e.g., betweenness relations in models for decision making [25] and aggregation [24], ternary order relation in string matching [16], cyclic orders in qualitative spatial reasoning [15] and particular fuzzy ternary relations in models of choice behavior [22].

In the ternary setting, the notion of transitivity has received far less attention. It has appeared in few papers, for instance, Pitcher and Smiley [26] have defined several notions of four point transitivity and five point transitivity of a betweenness relation. Also, Novák and Novotný [20], Chajda et al. [7], Barkat et al. [2] and Zedam et al. [29] have defined other forms of transitivity of a ternary relation.

Motivated by the increasing importance of ternary relations and the usefulness of the transitivity property of (fuzzy) binary relations, in this note we study the transitivity property for ternary fuzzy relations. In order to investigate this notion analogously to that of (fuzzy) binary relations, we extend the six types of composition of crisp ternary relations introduced by Bakri et al. [1] to the fuzzy setting. Based on these compositions of ternary fuzzy relations, we introduce several types of transitivity and investigate their representation and their properties.

This note is organized as follows. In Section 2, we recall the necessary concepts of lattices, t-norms and ternary fuzzy relations. In Section 3, we extend the compositions of ternary relations to ternary fuzzy relations and investigate their basic properties. In Section 4 , we introduce several types of transitivity of a 
ternary fuzzy relation and show several basic properties. Also, we study the interaction of these transitivity properties with the binary projections and cylindrical extensions, and we provide a representation of a given transitive ternary fuzzy relation in terms of its projections and a family of functions. Finally, we present some concluding remarks in Section 5.

\section{Preliminaries}

This section serves introductory purposes. First, we recall some basic concepts related to lattices and tnorms. Second, we present some basic definitions related to ternary fuzzy relations.

\section{$2.1 \quad$ Lattices}

A poset $(L, \leq)$ (see, e.g., [8]) is called a lattice if any two elements $x$ and $y$ have a greatest lower bound, denoted $x \wedge y$ and called the meet (infimum) of $x$ and $y$, as well as a smallest upper bound, denoted $x \vee y$ and called the join (supremum) of $x$ and $y$. A lattice can also be defined as an algebraic structure, namely a set $L$ equipped with two binary operations $\wedge$ and $\checkmark$ that are idempotent, commutative and associative, and satisfy the absorption laws $(x \wedge(x \vee y)=x$ and $x \vee(x \wedge y)=x$, for any $x, y \in L)$. The order relation and the meet and join operations are related as follows: $x \leq y$ if and only if $x \wedge y=x ; x \leq y$ if and only if $x \vee y=$ $y$. A bounded lattice is a lattice that additionally has a greatest element 1 and a smallest element 0 , which satisfy $0 \leq x \leq 1$ for every $x$ in $L$. Often, the notation $(L, \wedge, \vee, 0,1)$ is used. A lattice $(L, \wedge, \vee)$ is called a complete lattice if any nonempty subset of $L$ has an infimum and a supremum. The corresponding infimum and supremum operations are denoted as $\Lambda$ and $\bigvee$, respectively.

A triangular norm (t-norm, for short) $*$ on a bounded lattice $L$ (see, e.g., $[9,10])$ is a binary operation on $L$ that is commutative (i.e., $x * y=y * x$, for any $x, y \in L$ ) and associative (i.e., $x *(y * z)=(x * y) * z$, for any $x, y, z \in L$ ), has neutral element 1 (i.e., $x * 1=x$, for any $x \in L$ ) and is order-preserving (i.e., if $x \leq y$, then $x * z \leq y * z$, for any $x, y, z \in L)$. T-norms were originally introduced on the real unit interval $[0,1]$, but are readily extended to posets and lattices [10].

Throughout this paper, $L$ always denotes a complete lattice $(L, \wedge, \vee, 0,1)$ and $*$ a t-norm on it.

\subsection{Ternary fuzzy relations}

The notion of an $L$-fuzzy relation on a set $X$ generalizes the classical notion of a $\{0,1\}$-relation by expressing degrees of relationship in some bounded lattice $(L, \wedge, \vee, 0,1)[12]$.
Definition 2.1. A ternary (or triadic) $L$-fuzzy relation (ternary $L$-relation, for short) $T$ on $X$ is an $L$ fuzzy subset of $X^{3}$, i.e., a mapping $T: X^{3} \rightarrow L$. If $L=\{0,1\}$, then ternary relations are obtained.

Inclusion, intersection and union of ternary $L$-relations on $X$ are defined through the corresponding notions for $L$-fuzzy subsets of $X^{3}$. We denote by $T^{t}$ the transpose of $T$, i.e., for any $x, y, z \in X, T^{t}(x, y, z)=$ $T(z, y, x)$. For more details on (fuzzy) ternary relations, we refer to $[1,7,19,22,29]$.

\section{Compositions of ternary fuzzy relations}

In this section, we extend the types of composition of crisp ternary relations introduced in [1] to the fuzzy setting, and investigate their properties.

Definition 3.1. [1] Let $T$ and $S$ be two ternary relations on a set $X$. The $\circ_{i}$-compositions of $T$ and $S$, with $i \in\{1, \ldots, 6\}$, are defined as

(i) $T \circ_{1} S=\left\{(x, y, z) \in X^{3} \mid(\exists t, s \in X)((x, y, t) \in\right.$ $T \wedge(s, t, z) \in S)\}$

(ii) $T \circ_{2} S=\left\{(x, y, z) \in X^{3} \mid(\exists t, s \in X)((x, y, t) \in\right.$ $T \wedge(t, s, z) \in S)\}$

(iii) $T \circ_{3} S=\left\{(x, y, z) \in X^{3} \mid(\exists t, s \in X)((x, y, t) \in\right.$ $T \wedge(t, z, s) \in S)\}$;

(iv) $T \circ_{4} S=\left\{(x, y, z) \in X^{3} \mid(\exists t, s \in X)((s, x, t) \in\right.$ $T \wedge(t, y, z) \in S)\}$

(v) $T \circ_{5} S=\left\{(x, y, z) \in X^{3} \mid(\exists t, s \in X)((x, s, t) \in\right.$ $T \wedge(t, y, z) \in S)\}$

(vi) $T \circ_{6} S=\left\{(x, y, z) \in X^{3} \mid(\exists t, s \in X)((x, s, t) \in\right.$ $T \wedge(s, y, z) \in S)\}$.

Definition 3.2. Let $T$ and $S$ be two ternary $L$ relations on a set $X$. The $\circ_{i}$-compositions of $T$ and $S$, with $i \in\{1, \ldots, 6\}$, are defined as

(i) $T \circ_{1} S(x, y, z)=\bigvee_{s, t \in X}(T(x, y, t) * S(s, t, z))$;

(ii) $T \circ_{2} S(x, y, z)=\bigvee_{s, t \in X}(T(x, y, t) * S(t, s, z))$;

(iii) $T \circ_{3} S(x, y, z)=\bigvee_{s, t \in X}(T(x, y, t) * S(t, z, s))$;

(iv) $T \circ_{4} S(x, y, z)=\bigvee_{s, t \in X}(T(s, x, t) * S(t, y, z))$;

(v) $T \circ_{5} S(x, y, z)=\bigvee_{s, t \in X}(T(x, s, t) * S(t, y, z))$; 
(vi) $T \circ_{6} S(x, y, z)=\bigvee_{s, t \in X}(T(x, s, t) * S(s, y, z))$.

More generally, other types of composition of ternary $L$-relations can be obtained by combining the above six types of composition, e.g. as follows:

$$
T \circ_{I} S=\bigcup_{i \in I} T \circ_{i} S,
$$

for any $I \subseteq\{1, \ldots, 6\}$.

Next, we investigate some properties of the compositions of ternary $L$-relations. First, the following proposition shows that for any $i \in\{1,2,5,6\}$, the $\circ_{i^{-}}$ composition is associative.

Proposition 3.3. Let $T_{1}, T_{2}$ and $T_{3}$ be ternary $L$ relations on a set $X$. Then it holds that

$$
\left(T_{1} \circ_{i} T_{2}\right) \circ_{i} T_{3}=T_{1} \circ_{i}\left(T_{2} \circ_{i} T_{3}\right),
$$

for any $i \in\{1,2,5,6\}$.

In the following example, we show that the compositions $\circ_{3}$ and $\circ_{4}$ are not associative.

Example 3.4. Le $X=\{a, b, c, d\}, L=[0,1]$ and $*=$ $\wedge$. Let $T_{1}, T_{2}$ and $T_{3}$ be the ternary $L$-relations on $X$ given by:

$$
\begin{aligned}
& T_{1}(x, y, z)= \begin{cases}0.7 & , \text { if }(x, y, z)=(a, a, b) \\
0.5 & , \text { if }(x, y, z)=(a, b, c) \\
0 & , \text { otherwise }\end{cases} \\
& T_{2}(x, y, z)= \begin{cases}0.9 & , \text { if }(x, y, z)=(a, a, a) \\
0.65 & , \text { if }(x, y, z)=(b, d, a) \\
0.4 & \text {, if }(x, y, z)=(c, b, b) \\
0 & , \text { otherwise }\end{cases} \\
& T_{3}(x, y, z)= \begin{cases}0.8 & , \text { if }(x, y, z)=(a, a, a) \\
0.6 & , \text { if }(x, y, z)=(d, d, b) \\
0.25 & , \text { if }(x, y, z)=(b, c, a) \\
0 & , \text { otherwise }\end{cases}
\end{aligned}
$$

One easily verifies that

$$
\begin{aligned}
& \left(T_{1} \circ_{3} T_{2}\right) \circ_{3} T_{3}(x, y, z)= \begin{cases}0.6 & , \text { if }(x, y, z)=(a, a, d) \\
0.25 & , \text { if }(x, y, z)=(a, b, c) \\
0 & , \text { otherwise }\end{cases} \\
& T_{1} \circ_{3}\left(T_{2} \circ_{3} T_{3}\right)(x, y, z)= \begin{cases}0.65 & , \text { if }(x, y, z)=(a, a, d) \\
0.25 & , \text { if }(x, y, z)=(a, b, b) \\
0 & , \text { otherwise }\end{cases}
\end{aligned}
$$

It is clear that

$$
\left(T_{1} \circ_{3} T_{2}\right) \circ_{3} T_{3} \neq T_{1} \circ_{3}\left(T_{2} \circ_{3} T_{3}\right) .
$$

In the same line, one can also verify that

$$
\left(T_{1} \circ_{4} T_{2}\right) \circ_{4} T_{3} \neq T_{1} \circ_{4}\left(T_{2} \circ_{4} T_{3}\right) \text {. }
$$

The following proposition shows the interaction of the $\circ_{i}$-composition with inclusion and set-theoretical operations, for any $i \in\{1, \ldots, 6\}$.

Proposition 3.5. Let $T_{1}, T_{2}, S_{1}, S_{2}$ and $S$ be ternary $L$-relations on a set $X$. For any $i \in\{1, \ldots, 6\}$, the following statements hold:

(i) If $T_{1} \subseteq T_{2}$ and $S_{1} \subseteq S_{2}$, then $T_{1} \circ_{i} S_{1} \subseteq T_{2} \circ_{i} S_{2}$;

(ii) $\left(T_{1} \cap T_{2}\right) \circ_{i} S=\left(T_{1} \circ_{i} S\right) \cap\left(T_{2} \circ_{i} S\right)$ and $S \circ_{i}\left(T_{1} \cap\right.$ $\left.T_{2}\right)=\left(S \circ_{i} T_{1}\right) \cap\left(S \circ_{i} T_{2}\right)$;

(iii) $\left(T_{1} \cup T_{2}\right) \circ_{i} S=\left(T_{1} \circ_{i} S\right) \cup\left(T_{2} \circ_{i} S\right)$ and $S \circ_{i}\left(T_{1} \cup\right.$ $\left.T_{2}\right)=\left(S \circ_{i} T_{1}\right) \cup\left(S \circ_{i} T_{2}\right)$

Combining Propositions 3.3 and 3.5 leads to the following corollary.

Corollary 3.6. The compositions $\circ_{I}$ with $I \subseteq$ $\{1,2,5,6\}$ are associative.

The following proposition shows the interaction of the ${ }{ }_{I}$-composition with inclusion and set-theoretical operations, for any $I \subseteq\{1, \ldots, 6\}$.

Proposition 3.7. Let $T_{1}, T_{2}, S_{1}, S_{2}$ and $S$ be ternary $L$-relations on a set $X$. For any $I \subseteq\{1, \ldots, 6\}$, the following statements hold:

(i) If $T_{1} \subseteq T_{2}$ and $S_{1} \subseteq S_{2}$, then $T_{1} \circ_{I} S_{1} \subseteq T_{2} \circ_{I} S_{2}$;

(ii) $\left(T_{1} \cap T_{2}\right) \circ_{I} S \subseteq\left(T_{1} \circ_{I} S\right) \cap\left(T_{2} \circ_{I} S\right)$;

(iii) $\left(T_{1} \cup T_{2}\right) \circ_{I} S \subseteq\left(T_{1} \circ_{I} S\right) \cup\left(T_{2} \circ_{I} S\right)$.

\section{Transitivity of ternary fuzzy relations}

In this section, based on the compositions of ternary fuzzy relations, we introduce several types of transitivity of a ternary fuzzy relation and investigate their properties.

\subsection{Definitions and basic properties}

For a given binary $L$-relation $R$ on a set $X$, the *transitivity is defined as: $R(x, y) * R(y, z) \leq R(x, z)$, for any $x, y, z \in X$. This property is equivalent to the condition that $R \circ R \subseteq R$, where $\circ$ is the sup*-composition of binary $L$-relations, i.e., for any two binary $L$-relations $R_{1}$ and $R_{2}$ on $X$ :

$$
R_{1} \circ R_{2}(x, z)=\bigvee_{y \in X}\left(R_{1}(x, y) * R_{2}(y, z)\right) .
$$

Analogously to the binary case, we introduce the notions of transitivity of a ternary $L$-relation based on the sup-*-compositions of ternary $L$-relations introduced above. 
Definition 4.1. Let $T$ be a ternary $L$-relation on a set $X . T$ is called $\circ_{i}$-transitive if $T \circ_{i} T \subseteq T$, for any $i \in\{1, \ldots, 6\}$. This condition corresponds to

(i) $\circ_{1}$-transitive, if, for any $x, y, z, s, t \in X$, it holds that $T(x, y, t) * T(s, t, z) \leq T(x, y, z)$;

(ii) $\circ_{2}$-transitive, if, for any $x, y, z, s, t \in X$, it holds that $T(x, y, s) * T(s, t, z) \leq T(x, y, z)$;

(iii) $\circ_{3}$-transitive, if, for any $x, y, z, s, t \in X$, it holds that $T(x, y, s) * T(s, t, z) \leq T(x, y, t)$;

(iv) $\circ_{4}$-transitive, if, for any $x, y, z, s, t \in X$, it holds that $T(x, y, s) * T(s, t, z) \leq T(y, t, z)$;

(v) $\circ_{5}$-transitive, if, for any $x, y, z, s, t \in X$, it holds that $T(x, y, s) * T(s, t, z) \leq T(x, t, z)$;

(vi) $\circ_{6}$-transitive, if, for any $x, y, z, s, t \in X$, it holds that $T(x, s, t) * T(s, y, z) \leq T(x, y, z)$.

For a given ternary $L$-relation, other types of transitivity can be introduced as follows:

$T$ is called $\circ_{I}$-transitive if $T \circ_{I} T \subseteq T$, where $I \subseteq$ $\{1, \ldots, 6\}$.

One can easily verify that $T$ is called $\circ_{I}$-transitive if it is $\circ_{i}$-transitive, for any $i \in I \subseteq\{1, \ldots, 6\}$.

The following proposition shows that for any $i \in$ $\{1, \ldots, 6\}$, the $\circ_{i}$-transitivity is preserved under intersection.

Proposition 4.2. Let $\left(T_{j}\right)_{j \in J}$ be a family of ternary $L$-relations on a set $X$. For any $i \in\{1, \ldots, 6\}$, it holds that if $T_{j}$ is $\circ_{i}$-transitive for any $j \in J$, then $\underset{j \in J}{\cap} T_{j}$ is $o_{i}$-transitive.

Proposition 4.3. Let $T$ be a ternary L-relation on a set $X$. For any $i \in\{1, \ldots, 6\}$, it holds that $T$ is $\circ_{i}$-transitive if and only if $T^{t}$ is $\circ_{7-i}$-transitive.

Note that the $o_{I}$-transitivity of $\left(T_{j}\right)_{j \in J}$ implies the ${ } I^{-}$-transitivity of $\underset{j \in J}{\cap} T_{j}$. Also, the $\circ_{I}$-transitivity of $T$ is equivalent to the ${ }^{7}-I_{\text {-transitivity of }} T^{t}$, where $7-I=\{7-i \mid i \in I\}$, for any $I \subseteq\{1, \ldots, 6\}$.

The following proposition expresses that any ternary $L$-relation is the union of $\circ_{i}$-transitive ternary $L$ subrelations.

Proposition 4.4. Any ternary L-relation on a set $X$ is the union of $\circ_{i}$-transitive ternary $L$-subrelations on $X$, for any $i \in\{1, \ldots, 6\}$.

Remark 4.5. In the finite case, maximal $\circ_{i}$-transitive $L$-subrelations (in the sense of inclusion) of a given ternary $L$-relation can be obtained.

\subsection{Interaction of the types of transitivity with the projections and cylindrical extensions}

In this subsection, we discuss the interaction of several types of transitivity of a ternary $L$-relation with the binary projections and the cylindrical extensions. First, we recall the following definitions.

Definition 4.6. Let $T$ be a ternary $L$-relation on a set $X$.

(i) The left projection of $T$ with respect to $z \in X$ is the binary $L$-relation ${ }_{z} T$ on $X$ defined as

$$
{ }_{z} T(x, y)=T(z, x, y)
$$

(ii) The middle projection of $T$ with respect to $z \in X$ is the binary $L$-relation $T_{z}$ on $X$ defined as

$$
\underset{z}{T}(x, y)=T(x, z, y)
$$

(iii) The right projection of $T$ with respect to $z \in X$ is the binary $L$-relation $T_{z}$ on $X$ defined as

$$
T_{z}(x, y)=T(x, y, z) \text {. }
$$

Definition 4.7. Let $T$ be a ternary $L$-relation on a set $X$.

(i) The left projection of $T$ is the binary $L$-relation $P_{\ell}^{\vee}(T)$ on $X$ defined as

$$
P_{\ell}(T)(x, y)=\bigvee_{z \in X}{ }_{z} T(x, y) ;
$$

(ii) The middle projection of $T$ is the binary $L$ relation $P_{m}^{\vee}(T)$ on $X$ defined as

$$
P_{m}(T)(x, y)=\bigvee_{z \in X} T(x, y) ;
$$

(iii) The right projection of $T$ is the binary $L$-relation $P_{r}^{\vee}(T)$ on $X$ defined as

$$
P_{r}(T)(x, y)=\bigvee_{z \in X} T_{z}(x, y) .
$$

Definition 4.8. Let $R$ be a binary $L$-relation on a set $X$.

(i) The left cylindrical extension of $R$ is the ternary $L$-relation $C_{\ell}(R)$ on $X$ defined as

$$
C_{\ell}(R)(x, y, z)=R(y, z) ;
$$

(ii) The middle cylindrical extension of $R$ is the ternary relation $C_{m}(R)$ on $X$ defined as

$$
C_{m}(R)(x, y, z)=R(x, z) ;
$$


(iii) The right cylindrical extension of $R$ is the ternary relation $C_{r}(R)$ on $X$ defined as

$$
C_{r}(R)(x, y, z)=R(x, y) .
$$

The following proposition expresses the interaction of the $o_{i}$-transitivity of a given ternary $L$-relation with the $*$-transitivity of its binary projections.

Proposition 4.9. Let $T$ be a ternary $L$-relation on a set $X$. The following implications hold:

(i) If $T$ is $\circ_{1}$-transitive, then $P_{\ell}(T)$ is *-transitive;

(ii) If $T$ is $\mathrm{O}_{2}$-transitive, then $P_{m}(T)$ is $*$-transitive;

(iii) If $T$ is $\circ_{5}$-transitive, then $P_{m}(T)$ is $*$-transitive;

(iv) If $T$ is $\circ_{6}$-transitive, then $P_{r}(T)$ is *-transitive.

The following proposition discusses the interaction of the $*$-transitivity of a given binary $L$-relation with the ${ }^{\circ}{ }_{i}$-transitivity of its cylindrical extensions.

Proposition 4.10. Let $R$ be a binary L-relation on a set $X$. The following equivalences hold:

(i) $R$ is *-transitive if and only if $C_{\ell}(R)$ is $\circ_{1}$ transitive;

(ii) $R$ is *-transitive if and only if $C_{m}(R)$ is $\circ_{2}$ transitive;

(iii) $R$ is *-transitive if and only if $C_{m}(R)$ is $\mathrm{O}_{5-}^{-}$ transitive;

(iv) $R$ is *-transitive if and only if $C_{r}(R)$ is ${ }^{6^{-}}$ transitive.

\subsection{Representation of $\circ_{i}$-transitive ternary fuzzy relations}

In this subsection, we provide a representation theorem for $\circ_{i}$-transitive ternary $L$-relations, for any $i \in$ $\{1,2,5,6\}$. First, we need to recall the following definition of implication generated by a t-norm on a complete lattice. For more details on implications, see, e.g., Bělohlávek [4].

Let $*$ be a t-norm on a complete lattice $L$. Then the $*$-residuum (or the $*$-implication) is defined for any $x, y \in L$ by:

$$
I_{*}(x, y)=\bigvee\{z \in L \quad \mid x * z \leq y\} .
$$

In the following representation theorems for $\circ_{i^{-}}$ transitive (resp. reflexive and $\circ_{i}$-transitive) ternary $L$ relations with $i \in\{1,2,5,6\}$, we consider only t-norms that satisfy the following condition:

$$
x * z \leq y \Leftrightarrow z \leq I_{*}(x, y),
$$

for any $x, y, z \in L$. Equivalently, one can suppose that $\left(L, \wedge, \vee, *, I_{*}, 0,1\right)$ is a complete residuated lattice. For instance, on the unit interval, this condition characterizes left-continuous t-norms, including minimum, product and the Eukasiewicz t-norm.

Theorem 4.11. Let $T$ be a ternary L-relation on a set $X$. The following equivalences hold:

(i) $T$ is $\circ_{1}$-transitive if and only if there exist a family $\left(f_{a}\right)_{a \in X}$ of functions from $X$ to $L$ such that $T_{a}(x, y) \leq f_{a}(y)$, for any $x, y, a \in X$ and

$$
T(x, y, z)=\bigwedge_{a \in X} I_{*}\left(f_{a}(z), T_{a}(x, y)\right),
$$

for any $x, y, z \in X$

(ii) $T$ is $\mathrm{O}_{2}$-transitive if and only if there exist a family $\left(f_{a}\right)_{a \in X}$ of functions from $X$ to $L$ such that $T_{a}(x, y) \leq f_{a}(x)$, for any $x, y, a \in X$ and

$$
T(x, y, z)=\bigwedge_{a \in X} I_{*}\left(f_{a}(z), T_{a}(x, y)\right),
$$

for any $x, y, z \in X$

(iii) $T$ is $\circ_{5}$-transitive if and only if there exist a family $\left(f_{a}\right)_{a \in X}$ of functions from $X$ to $L$ such that ${ }_{a} T(x, y) \leq f_{a}(y)$, for any $x, y, a \in X$ and

$$
T(x, y, z)=\bigwedge_{a \in X} I_{*}\left(f_{a}(x),{ }_{a} T(y, z)\right),
$$

for any $x, y, z \in X$;

(iv) $T$ is $\circ_{6}$-transitive if and only if there exist a family $\left(f_{a}\right)_{a \in X}$ of functions from $X$ to $L$ such that ${ }_{a} T(x, y) \leq f_{a}(x)$, for any $x, y, a \in X$ and

$$
T(x, y, z)=\bigwedge_{a \in X} I_{*}\left(f_{a}(x),{ }_{a} T(y, z)\right),
$$

for any $x, y, z \in X$.

\section{Conclusion}

In this note, we have extended the types of composition of ternary relations to the fuzzy setting. We have introduced several types of transitivity of a ternary fuzzy relation close in spirit to the $*$-transitivity of a binary fuzzy relation. We have investigated their properties, and their interaction with the binary projections of ternary fuzzy relations and cylindrical extensions of binary fuzzy relations. Moreover, we have provided a representation of transitive (resp. reflexive and transitive) ternary fuzzy relations for four types of transitivity. Consequently, the representation obtained can be applied to represent some important classes of ternary fuzzy relations, like fuzzy betweenness relations and ternary fuzzy equivalence relations. 


\section{References}

[1] N. Bakri, L. Zedam, B. De Baets, Compositions of ternary relations, Information and computation.

[2] O. Barkat, L. Zedam and B. De Baets, On the compatibility of a ternary relation with a binary fuzzy relation, International Journal of Uncertainty, Fuzziness and Knowledge-Based Systems (IJUFKS) 27 (2019).

[3] J. Beall, R. Brady, J.M. Dunn, A.P. Hazen, E. Mares, R.K. Meyer, G. Priest, G. Restall, D. Ripley, J. Slaney, R. Sylvan, On the ternary relation and conditionality, Journal of Philosophical Logic 41 (2012) 595-612.

[4] R. Bělohlávek, Fuzzy Relational Systems: Foundations and Principles, Kluwer Academic Publishers/Plenum Publishers, New York, 2002.

[5] R. Bělohlávek, P. Osicka, Triadic fuzzy Galois connections as ordinary connections, Fuzzy Sets and Systems 249 (2014) 83-99.

[6] H. Borzecka, Multi-criteria decision making using fuzzy preference relations, Operations Research and Decisions 14 (2012) 5-21.

[7] I. Chajda, M. Kolařík, H. Länger, Algebras assigned to ternary relations, Miskolc Mathematical Notes 14 (2013) 827-844.

[8] B.A. Davey, H.A. Priestley, Introduction to Lattices and Order, Cambridge University Press, 2nd ed, Cambridge, 2002.

[9] B. De Baets, R. Mesiar, Triangular norms on product lattices, Fuzzy Sets and Systems 104 (1999) 61-75.

[10] G. De Cooman, E.E. Kerre, Order norms on bounded partially ordered sets, Journal of Fuzzy Mathematics 2 (1994) 281-310.

[11] J. Fodor, M. Roubens, Fuzzy Preference Modelling and Multicriteria Decision Support, Kluwer Academic Publishers, Dordrecht, 1994.

[12] J.A. Goguen, L-fuzzy sets, Journal of Mathematical Analysis and Applications 18 (1967) 145-174.

[13] Z.W. Gong, Y. Lin, T.X. Yao, Uncertain Fuzzy Preference Relations and Their Applications, Springer-Verlag, Berlin, Heidelberg, 2013.

[14] D.I. Ignatov, D.V. Gnatyshak, S.O. Kuznetsov, B.G. Mirkin, Triadic formal concept analysis and triclustering: searching for optimal patterns, Machine Learning 101 (2015) 271-302.
[15] A. Isli, A. Cohn, A new approch to cyclic ordering of $2 \mathrm{D}$ orientations using ternary relation algebra, Artificial Intelligence 122 (2000) 137-187.

[16] J. Kim, A. Amir, J.C. Na, K. Park, J.S. Sim, On representations of ternary order relations in numeric strings, Mathematics in Computer Science 11 (2017) 127-136.

[17] F. Klawonn, R. Kruse, Equality relations as a basis for fuzzy control, Fuzzy Sets and Systems 54 (1993) 147-156.

[18] J. Konečný, P. Osička, Triadic concept lattices in the framework of aggregation structures, Information Sciences 279 (2014) 512-527.

[19] V. Novák, M. Novotný, Pseudodimension of relational structures, Czechoslovak Mathematical Journal 49 (1999) 547-560.

[20] V. Novák, M. Novotný, On representation of cyclically ordered sets, Czechoslovak Mathematical Journal 39 (1989) 127-132.

[21] T. Onishi, Understanding negation implicationally in the relevant Logic R, Studia Logica 104 (2016) 1267-1285.

[22] S. Ovchinnikov, Decision Making with Fuzzy Ternary Relations, in Modern Information Processing: From Theory to Applications, (B. Bouchon-Meunier, G. Coletti and R. Yager, eds.), Elsevier Science, 2006, pp. 111-119.

[23] S. Ovchinnikov, On the transitivity property, Fuzzy Sets and Systems 20 (1986) 241-243.

[24] R. Pérez-Fernández, B. De Baets, On the role of monometrics in penalty-based data aggregation, IEEE Transactions on Fuzzy Systems, DOI: 10.1109/TFUZZ.2018.2880716.

[25] R. Pérez-Fernández, M. Rademaker, B. De Baets, Monometrics and their role in the rationalisation of ranking rules, Information Fusion 34 (2017) 1627.

[26] E. Pitcher, M.F. Smiley, Transitivities of betweenness, Transactions of the American Mathematical Society 52 (1942) 95-114.

[27] S. Powers, Practical RDF, O'Reilly, Beijing, 2003.

[28] M. Steel, Phylogeny: Discrete and Random Processes in Evolution, PA: SIAM, Philadelphia, 2016.

[29] L. Zedam, O. Barkat, B. De Baets, Traces of ternary relations, International Journal of General Systems 47 (2018) 350-373. 